\title{
Electric-field controlled spin in bilayer triangular graphene quantum dots
}

\author{
A. D. Güçlü, ${ }^{1}$ P. Potasz, ${ }^{1,2}$ and P. Hawrylak ${ }^{1}$ \\ ${ }^{1}$ Institute for Microstructural Sciences, National Research Council of Canada, Ottawa, Canada \\ ${ }^{2}$ Institute of Physics, Wroclaw University of Technology, Wroclaw, Poland
}

(Dated: November 7, 2018)

\begin{abstract}
We present theoretical results based on mean-field and exact many-body approaches showing that in bilayer triangular graphene quantum dots with zigzag edges the magnetism can be controlled by an external vertical electric-field. We demonstrate that without electric field the spins of the two layers of the quantum dot interact ferromagnetically. At a critical value of the electric-field, the total spin of the bilayer structure can be turned off or reduced to a single localized spin, a qubit isolated from contacts and free from interaction with nuclear spins.
\end{abstract}

Graphene exhibits unusual electronic properties $1-6$ including relativistic nature of quasi-particles, sublattice pseudospin, and zero energy bandgap. The application of graphene for logic devices requires opening of the bandgap which can be achieved by either size quantization [7 18], or chemical modification [19], or bringing in a second layer and applying an external electricfield 20 28], or size quantization in bilayer graphene nanostructures 29 32]. In particular, when graphene is reduced to a triangular quantum dot with zigzag edges [8], sublattice symmetry is broken, size dependent energy gap is open, and a band of degenerate states at the Fermi level is created leading to finite macroscopic spin polarization [11 18]. This allows simultaneous size, shape and edge engineering of magnetic, electrical, and optical properties in a single material-graphene 14].

In this work, we investigate electronic and magnetic properties of bilayer triangular graphene quantum dots with zigzag edges under external vertical electric-field. We show that the magnetic moment of bilayer triangular graphene quantum dots can be controlled by the vertical electric-field. Without the electric-field, the magnetic moments of the two layers are shown to be coupled ferromagnetically. Using configuration interaction and mean-field calculations based on tight-binding model, we demonstrate that the ferromagnetism can be either turned off or reduced to a single electron/hole spin. The single electron spin is hence isolated in a charge neutral structure by the application of an electric-field, independent of the size of the quantum dot and without decoherence due to contacts. The electric-field control of the ferromagnetism [33] and isolation of a single spin opens new applications in spintronics and quantum information processing 34 38].

Figure 1a shows two possibilities for building a bilayer triangular graphene quantum dot (BQD) using two single layer triangular quantum dots (TGQD) of comparable sizes, with zigzag edges. We consider AB Bernal stacking, where the A sublattice of the top layer (A2, shown in blue color) is on top of the B sublattice of the bottom layer (B1, shown in red). On the left hand side, the two TGQDs are of the same size. In this configuration,
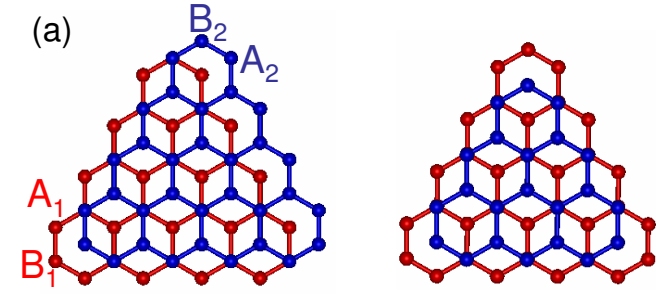

(b)
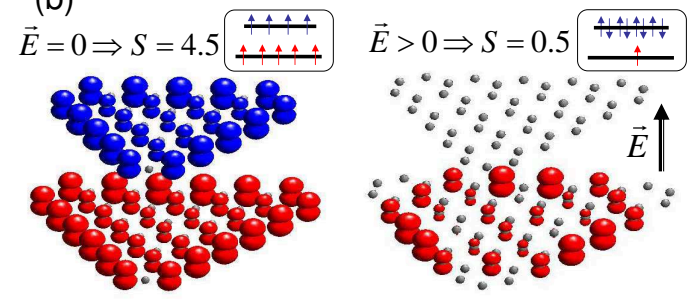

FIG. 1: (Color online) Bilayer triangular graphene quantum dot with zigzag edges. (a) structure constructed using two single layer quantum dots with equal sizes (on the left) and different sizes (on the right). (b) Isosurface plot of the spin density $\rho_{\uparrow}-\rho_{\downarrow}$ for with and without electric-field $\mathbf{E}$, obtained from configuration interaction calculations.

however, not all the A2 atoms have a B1 partner as required by Bernal stacking. A more natural configuration choice is shown on the right hand side of Fig.1a. The top layer triangle has its floating atoms removed, making it smaller than the bottom layer triangle. Such a bilayer construction has the interesting property of having an odd number of degenerate states at the Fermi level (zero-energy shell of states) independent of its size, allowing to isolate a single spin in a charge neutral structure and hence isolated from the contacts. This is illustrated in Fig.1b, where the spin density isosurfaces are shown for zero electric-field (left hand side) and finite electric-field (right hand side), as obtained from our tight-binding based configuration interaction calculations explained in detail below. When the electric-field is off, both layers have a finite magnetic moment, as in single layer triangles [12 18], differing by one spin due to the size difference of the two triangles. The magnetic moments of the two layers are coupled ferromagnetically. When 

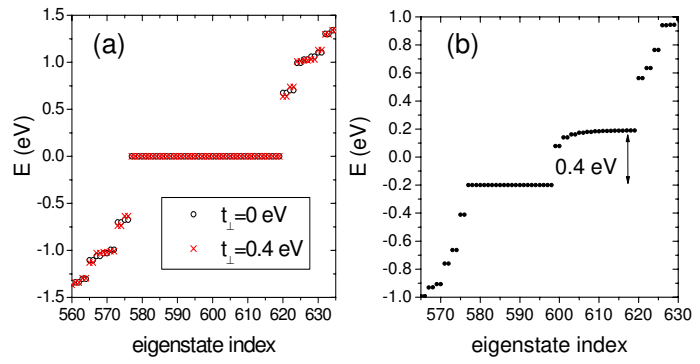

FIG. 2: (Color online) Single particle tight-binding spectrum. (a) The bilayer quantum dot consisting of 1195 atoms has 43 zero-energy states. (b) When an electric-field is applied, the degeneracy between the 21 top layer zero-energy states and 22 bottom layer zero-energy states is lifted.

a sufficiently high electric field is applied, electrons from the lower layer reduce their energy by flipping their spin and transfering to the top layer filling up all the available spin up and down zero-energy states, leaving behind one single spin.

We have confirmed the above picture through calculations with varying levels of accuracy. As a first step, we diagonalize the tight-binding Hamiltonian given by

$$
H_{T B}=\sum_{i j \sigma} \tau_{i j} c_{i \sigma}^{\dagger} c_{j \sigma}+\sum_{i \sigma} V_{i} c_{i \sigma}^{\dagger} c_{i \sigma}
$$

where the operator $c_{i \sigma}^{\dagger}$ creates an electron on a $p_{z}$ orbital on site $i$ with spin $\sigma$. The tight-binding parameters $\tau_{i j}$ are fixed to their bulk values $t=-2.8 \mathrm{eV}$ for in-plane nearest neighbours $i$ and $j$ and $t_{\perp}=0.4 \mathrm{eV}$ for inter-layer hopping. The effect of the potential difference induced by an external perpendicular electric-field $\mathbf{E}$ is taken into account through $V_{i}=-\Delta V / 2$ for the bottom layer atoms and $V_{i}=\Delta V / 2$ for the top layer atoms. Figure $2 \mathrm{a}$ shows the energy spectrum near the Fermi level for $\Delta V=0$ for a BQD consisting of 622 atoms in the bottom and 573 atoms in the top layer. If we take $t_{\perp}=0$, the two triangles are decoupled and we find 22 zero-energy states in the bottom layer and 21 zero-energy states in the top layer, for a total of 43 zero-energy states, consistent with previous work on single layer TGQDs 12 18]. Turning on $t_{\perp}$ to $0.4 \mathrm{eV}$ does not affect the zero-energy states. The effect of applying an electric field, e.g. $\Delta V=0.4$ $\mathrm{eV}$, is shown in Fig.2b. The energy of the 21 zero-energy states corresponding to the top layer is pushed up by 0.4 $\mathrm{eV}$ with respect to the bottom layer zero-energy states. Note that the bottom layer zero-energy states do not experience any dispersion unlike the top layer zero-energy states. This is due to the fact that they lie strictly on A1 sites which are not coupled to the top layer, whereas the top layer zero-energy states, which lie on B2, do couple to the bottom layer. The ability of controlling the relative position of zero-energy states gives an interesting opportunity to control the charge and spin of the zero-

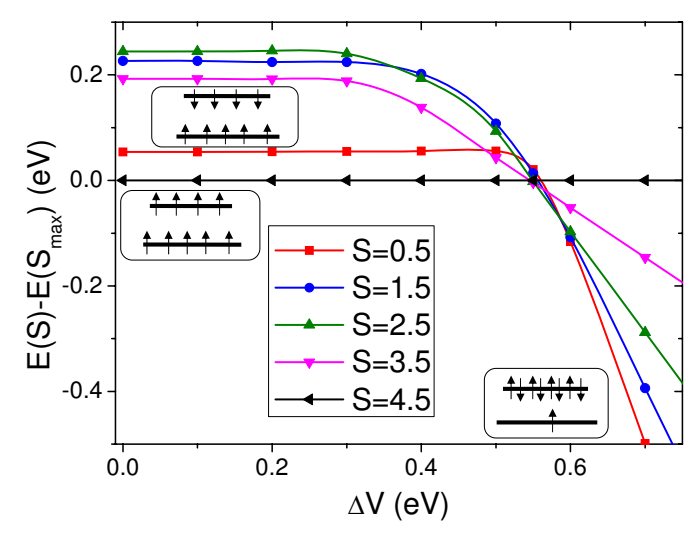

FIG. 3: (Color online) Meanfield Hubbard results. Energies of lowest energy states with different total spin $\mathrm{S}$ as a function of potential difference $\Delta V_{c}$ between the layers, with respect to the ferromagnetic configuration $S_{\max }=4.5$ for a system of 107 atoms and 9 zero-energy states.

energy states, expected to be spin polarized for $\Delta V=0$ according to Lieb's theorem 39].

The magnetic properties of BQD structures as a function of applied electric field can be studied by solving the Hubbard model within the self-consistent mean-field approach:

$H_{M F}=\sum_{i j \sigma} \tau_{i j} c_{i \sigma}^{\dagger} c_{j \sigma}+\sum_{i \sigma} V_{i} n_{i \sigma}+U \sum_{i \sigma}\left(\left\langle n_{i \sigma}\right\rangle-\frac{1}{2}\right) n_{i \sigma}(2)$

where $\mathrm{U}$ is the on-site Hubbard term taken to be 2.75 $\mathrm{eV}$ here. First, we study a small BQD of 107 atoms and 9 zero-energy states. Figure 3 shows the energies for different total spin $S$ with respect to the energy of the ferromagnetic configuration, $S=9 / 2$. At $\Delta V=0$, the degenerate band of zero-energy states is polarized: all 9 electrons occupying the 9 zero-energy states have their spins aligned ferromagnetically. Although the first excited state obtained from the Hubbard model is antiferromagnetic with the total spin of the bottom layer opposite to the total spin of the top layer, a full treatment of the correlation effects shows that (see below) low lying excited states have more complex spin structures. The Hubbard model is, however, useful for estimating the critical value $V_{c}$ where phase transition occurs. As $\Delta V$ is increased, the electrons lying on the bottom layer zeroenergy states are forced to flip their spin and tunnel to the top layer zero-energy states. At around $\Delta V_{c}=0.55$ $\mathrm{eV}$ such charge transfers occur abruptly, leading to a decrease of the total spin of the system. As a result, all top layer zero-energy states become doubly occupied, leaving exactly one single spin in the bottom layer zero-energy states. We note that one can also isolate a single hole spin in the bottom layer by applying a reverse electric field, thus pushing the electrons from the top layer to the bottom layer, occupying all states except one. It is thus possible to isolate and manipulate a single electron 

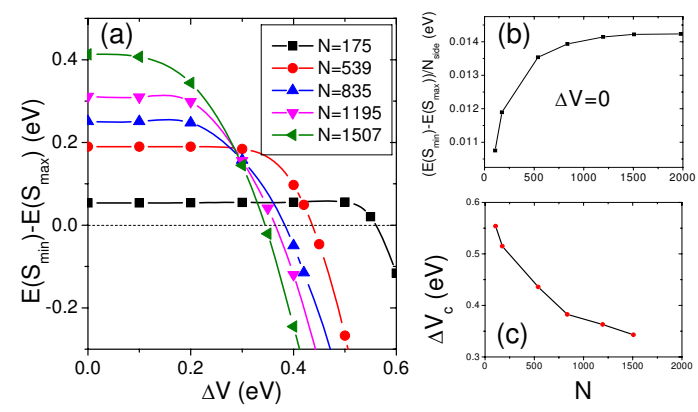

FIG. 4: (Color online) Size dependence of ferromagneticantiferromagnetic transition. (a) FM-AFM energy difference $E\left(S_{\min }\right)-E\left(S_{\max }\right)$ as a function of potential difference $\Delta V$ between layers, up to $N=1507$ atoms. (b) For $\Delta V=0$, the FM-AFM energy gap per number of side atoms $N_{\text {side }}$ approaches $14.3 \mathrm{meV}$ (top panel). Critical value $\Delta V_{c}$ where the transition occurs as a function of number of atoms $N$ (bottom panel).

or hole spin in a neutral BQD by applying an external electric field.

The procedure of isolating single electron or hole should occur regardless of the size of the system since the top layer has always one less zero-energy state than the bottom one. In order to investigate the size dependence, in Fig.4a we show the energy difference between the ferromagnetic and antiferromagnetic (FM-AFM) states calculated in the mean-field Hubbard approximation as function of applied voltage for several sizes up to 1507 atoms. We note that, due to the unusually high degeneracy of the states, self-consistent iterations occasionally get trapped in a local energy minima. We have thus repeated the calculations several times using different initial conditions and/or convergence schemes to assure that the correct ground state was reached. As expected, at $\Delta V=0$, the FM-AFM gap increases with the size of the system $N$. In fact, the FM-AFM gap energy per $N_{\text {side }}$, the number of side atoms on the top layer $\left(N_{\text {side }}\right)$ (equal the number of inter-layer bonds on the edges), approaches a constant value of $14.3 \mathrm{meV}$ as shown in Fig.4b. However, the FMAF transition voltage $V_{c}$ decreases with the system size as can be seen from Fig.4c. For the largest system size studied, $N=1507$, we obtain $\Delta V_{c}=0.345 \mathrm{eV}$, which corresponds to an electrical field of $\sim 1 \mathrm{~V} / \mathrm{nm}$, a value within experimental range 24].

We now test the predictions of the mean-field Hubbard model by including long range interaction and correlation effects, which is computationally feasible for the system size studied in Fig.3. We first solve a Hartree-Fock generalization of the Eq.2, but with empty zero-energy states [13]:
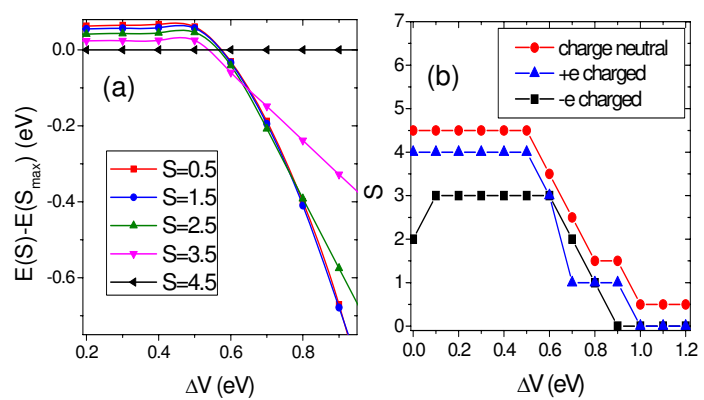

FIG. 5: (Color online) Configuration interaction results. (a) Energies of states for different total spin $S$ as a function of potential difference $\Delta V$ between the layers, with respect to the ferromagnetic configuration $S_{\max }=4.5$ for the same system as in Fig.3. (b) Ground state total spin $S$ as a function of $\Delta V$ for the charge neutral system, $-e$ charged system, and $+e$ charged system.

$$
\begin{gathered}
H_{H F}=\sum_{i j \sigma} \tau_{i j} c_{i \sigma}^{\dagger} c_{j \sigma}+\sum_{i \sigma} V_{i} n_{i \sigma}+\sum_{i l \sigma} \sum_{j k \sigma^{\prime}}\left(\rho_{j k \sigma^{\prime}}-\rho_{j k \sigma^{\prime}}^{b u l k}\right) \\
\times \quad\left(\left\langle i j\left|V_{e e}\right| k l\right\rangle-\left\langle i j\left|V_{e e}\right| l k\right\rangle \delta_{\sigma, \sigma^{\prime}}\right) c_{i \sigma}^{\dagger} c_{l \sigma}
\end{gathered}
$$

where the tight-binding term now includes the intralayer next-nearest neighbour hopping $t=-0.1 \mathrm{eV}$, interlayer next-nearest hoppings $\gamma_{3}=0.3 \mathrm{eV}$ and $\gamma_{4}=0.15$ $\mathrm{eV}[27]$. The terms $\rho$ and $\rho^{\text {bulk }}$ are quantum dot and bulk density matrices, respectively [13]. The two-body Coulomb matrix elements $\left\langle i j\left|V_{e e}\right| k l\right\rangle$ computed using Slater $p_{z}$ orbitals include on-site interactions, all scattering and exchange terms within next-nearest neighbors, and all long range direct interactions. We have previously tested the validity of our approach by comparing to density functional calculations and obtained good agreement[13]. After diagonalizing the Hartree-Fock Hamiltonian, we obtain Hartree-Fock quasi-particles denoted by the creation operator $b_{p \sigma}^{\dagger}$, with eigenvalues $\epsilon_{p}$ and eigenfunctions $|p\rangle$. We can now fill the new quasiparticle zero-energy states with electrons and solve the many-body Hamiltonian given by

$$
H=\sum_{p \sigma} \epsilon_{p} b_{p \sigma}^{\dagger} b_{p \sigma}+\frac{1}{2} \sum_{p q r s \sigma \sigma^{\prime}}\left\langle p q\left|V_{e e}\right| r s\right\rangle b_{p \sigma}^{\dagger} b_{q \sigma^{\prime}}^{\dagger} b_{r \sigma^{\prime}} b_{s \sigma}(4)
$$

Figure 5a shows the evolution of spin states for the same system studied in Fig.3, but now obtained by diagonalizing the many-body Hamiltonian. We observe two main differences from the mean-field Hubbard results: (i) At low $\Delta V$, the antiferromagnetic configuration $S=1 / 2$ is no longer the first excited state. Although the AFMFM energy gap is still comparable to the Hubbard result, other spin excitations are now closer to the ground state due to correlation effects. (ii) The ground state spin transitions do not occur as abruptly as in a Hubbard model. 
As shown in Fig.5b, the total spin of the charge neutral system (red color online, solid line with circles) evolves towards the minimum spin state $S=1 / 2$ gradually between $\Delta V=0.5-1.0 \mathrm{eV}$. This is mainly due to long range interactions. As the electrons are transferred one by one from the top layer into the bottom layer, they leave a positively charged hole behind which makes it harder to transfer more electrons. We note that this behavior of gradual spin transition is also obtained within a mean-field Hubbard model with long range interactions included (not shown). Finally, in Fig.5b, we also study the effect of charging the BQD system. The asymmetry between magnetic moment of $+e$ and $-e$ charged systems reflects the correlation induced spin depolarization process that occurs in single layer TGQDs as discussed in our previous works 13 .

In summary, we demonstrate that the graphene bilayer triangular quantum dots exhibit a shell of degenerate states at the Fermi level. At half filling, the shell is maximally spin polarized. By the application of a vertical electric field the total spin of the bilayer structure can be turned off or reduced to a single localized spin, a qubit isolated from contacts and free from interaction with nuclear spins. This opens new possibilities in the area of spintronics and quantum information processing.

Acknowledgment. The authors thank NRC-CNRS CRP, Canadian Institute for Advanced Research, Institute for Microstructural Sciences, and QuantumWorks for support.

[1] P. R. Wallace, Phys. Rev. 71 , 622 (1947).

[2] K. S. Novoselov, A. K. Geim, S. V. Morozov, D. Jiang, Y. Zhang, S. V. Dubonos, I. V. Grigorieva, and A. A. Firsov, Science 306, 666 (2004).

[3] K. S. Novoselov, A. K. Geim, S. V. Morozov, D. Jiang, M. I. Katsnelson, I. V. Grigorieva, S. V. Dubonos, and A. A. Firsov, Nature 438, 197 (2005).

[4] Y. B. Zhang, Y. W. Tan, H. L. Stormer, and P. Kim, Nature 438, 201 (2005).

[5] S. Y. Zhou, G. H. Gweon, J. Graf, A. V. Fedorov, C. D. Spataru, R. D. Diehl, Y. Kopelevich, D. H. Lee, S. G. Louie, and A. Lanzara, Nature Phys. 2, 595 (2006).

[6] A. H. C. Neto, F. Guinea, N. M. R. Peres, K. S. Novoselov, and A. K. Geim, Rev. of Mod. Phys. 81, 109 (2009).

[7] L. A. Ponomarenko, F. Schedin, M. I. Katsnelson, R. Yang, E. W. Hill, K. S. Novoselov, and A. K. Geim, Science 320, 356 (2008).

[8] Jiong Lu, Pei Shan Emmeline Yeo, Chee Kwan Gan, Ping $\mathrm{Wu}$ and Kian Ping Loh, Nature Nanotechnology 6, 247252 (2011).

[9] J. Guttinger, T. Frey, C. Stampfer, T. Ihn, and K. Ensslin, Phys. Rev. Lett. 105, 116801 (2010).

[10] B. Wunsch, T. Stauber, and F. Guinea, Phys. Rev. B 77, 035316 (2008).

[11] J. Akola, H. P. Heiskanen, and M. Manninen, Phys. Rev. B 77, 193410 (2008).

[12] M. Ezawa, Phys. Rev. B 81, 201402 (2010).
[13] A. D. Güçlü, P. Potasz, O. Voznyy, M. Korkusinski, and P. Hawrylak, Phys. Rev. Lett. 103, 246805 (2009).

[14] A. D. Güçlü, P. Potasz, and P. Hawrylak, Phys. Rev. B 82, 155445 (2010).

[15] P. Potasz, A. D. Güçlü, and P. Hawrylak, Phys. Rev. B 81, 033403 (2010).

[16] O. Voznyy, A.D. Güçlü, P. Potasz , P. Hawrylak, Phys.Rev.B. (in press).

[17] J. Fernandez-Rossier and J. J. Palacios, Phys. Rev. Lett. 99, 177204 (2007).

[18] W. L. Wang, S. Meng, and E. Kaxiras, Nano Letters 8, 241 (2008).

[19] D. C. Elias , R. R. Nair, T. M. G. Mohiuddin, S. V. Morozov, P. Blake, M. P. Halsall, A. C. Ferrari, D. W. Boukhvalov, M. I. Katsnelson, A. K. Geim and K. S. Novoselov, Science 323 610-613 (2009).

[20] E. McCann , D. S. L. Abergel, V. I. Falko, Solid St. Comm. 143, 110 (2007).

[21] T. Ohta, A. Bostwick, T. Seyller, K. Horn and E. Rotenberg, Science 313, 951 (2006).

[22] Eduardo V. Castro , K. S. Novoselov, S. V. Morozov, N. M. R. Peres, J. M. B. Lopes dos Santos, Johan Nilsson, F. Guinea, A. K. Geim, and A. H. Castro Neto, Phys. Rev. Lett. 99, 216802 (2007).

[23] J. B. Oostinga , Hubert B. Heersche, Xinglan Liu, Alberto F. Morpurgo and Lieven M. K. Vandersypen, Nat. Mater. 7, 151 (2007).

[24] K. F. Mak , C. H. Lui, J. Shan, and T. F. Heinz, Phys. Rev. Lett. 102, 256405 (2009).

[25] Y. Zhang T. T. Tang, C. Girit, Z. Hao, M. A. Martin, A. Zettl, M. F. Crommie, Y. R. Shen, F. Wang, Nature 459, 820 (2009).

[26] R. T. Weitz , M. T. Allen, B. E. Feldman, J. Martin and A. Yacoby, Science 330, 812 (2010).

[27] Eduardo V. Castro K. S. Novoselov, S. V. Morozov, N. M. R. Peres, J. M. B. Lopes dos Santos, Johan Nilsson, F. Guinea, A.K. Geim and A.H. Castro Neto, J. Phys.:Condens. Matter, 22, 175503 (2010).

[28] L.M. Zhang , Z. Q. Li, Dimitri N. Basov, M.M. Fogler, Zhao Hao, Michael C. Martin, Phys. Rev. B, 78, 235408 (2008).

[29] J. Milton Pereira, P. Vasilopoulos, and F. M. Peeters, Nano Lett., 7, 946 (2007).

[30] Eduardo V. Castro , N. M. R. Peres, J. M. B. Lopes dos Santos, A. H. Castro Neto, and F. Guinea, Phys. Rev. Lett. 100, 026802 (2008).

[31] Bhagawan Sahu, Hongki Min, A. H. MacDonald, and Sanjay K. Banerjee, Phys. Rev. B 78, 045404 (2008).

[32] A. R. Wright, J. C. Cao and C. Zhang, Phys. Rev. Lett. 103, 207401 (2009).

[33] H. Ohno D. Chiba, F. Matsukura, T. Omiya, E. Abe, T. Dietl1, Y. Ohno and K. Ohtani, Nature 408, 944 (2000).

[34] A. Rycerz, J. Tworzydo and C. W. J. Beenakker, Nature Physics 3, 172 (2007).

[35] Y. W. Son, M. L. Cohen, and S. G. Louie, Nature 444, 347 (2006)

[36] Bjrn Trauzettel Denis V. Bulaev, Daniel Loss and Guido Burkard, Nature Physics 3, 192 (2007).

[37] H. Sahin, R. T. Senger and S. Ciraci, J. Appl. Phys. 108, 074301 (2010).

[38] L. A. Agapito1, N. Kioussis1, and E. Kaxiras, Phys. Rev. B 82, 201411(R) (2010).

[39] E. H. Lieb, Phys. Rev. Lett. 62, 1201 (1989). 